\title{
Anos Potenciais de Vida Perdidos no Rio de Janeiro, 1990. As Mortes Violentas em Questão
}

\author{
Violent Death as a Cause of Years of Potential Life Lost in Rio de \\ Janeiro, 1990
}

\author{
Michael Eduardo Reichenheim ${ }^{1}$ \\ Guilherme L. Werneck' ${ }^{1}$
}

\begin{abstract}
REICHENHEIN, M. E. \& WERNECK, G. L. Violent Death as a Cause of Years of Potential Life Lost in Rio de Janeiro, 1990 . Cad. Saúde Públ., Rio de Janeiro, 10 (supplement 1): 188198, 1994.
\end{abstract}

This article is concerned with the magnitude of causes of death in the State and Municipality of Rio de Janeiro, in 1990, according to the "Years of Potential Life Lost" (YPLL) from one to 70 years of age. This indicator provides a more precise evaluation of the greater relevance of early deaths.

An analysis of YPLL by main causes of death shows that in both the State and Municipality, external causes (traumas and injuries) are the leading causes with over $30 \%$ of YPLL. This finding goes against the accepted mortality-rate-based view of chronic heart disease as the most important cause of death. Analysis of specific causes of death for both the State and Municipality puts homicides in first place, followed by cancers in the Municipality of Rio de Janeiro and "other types of violence" (suicides and violence of unknown type, whether accidental or intentional) in the State of Rio de Janeiro.

With the exception of cancers, YPLL rates by main death groups are consistently lower in the Municipality of Rio de Janeiro than the State as a whole, irrespective of gender. Absolute figures and rates for YPLL are strikingly higher in males, due mainly to "external causes". The authors stress the importance of using this indicator of premature deaths for defining priorities in health actions. The devastating effect of traumas and injuries as causes of deaths at early ages is also emphasized.

Key words: Trauma; Violence; Accidents, Suicide

\section{INTRODUÇÃO}

Nos últimos 30 anos, profundas transformações têm ocorrido no Brasil, em decorrência da industrialização e da crescente urbanização. Um novo perfil demográfico veio se desenhando com a queda progressiva e rápida da taxa de fecundidade nas regiões urbanas e rurais do país, com um correspondente aumento da esperança de vida e do índice de envelhecimento da população (Ramos et al., 1987).

${ }^{1}$ Instituto de Medicina Social da Universidade do Estado do Rio de Janeiro. Rua São Francisco Xavier, 524, $7^{\circ}$ andar, Rio de Janeiro, RJ, 20550-900, Brasil.
Associada a esta transformação demográfica ocorre uma mudança do quadro nosológico do país. Às doenças infecciosas somam-se as doenças cardiovasculares, as neoplasias e as causas externas, (o termo causas externas refere-se, de acordo com a $9^{\text {a }}$ Revisão da

Classificação Internacional de Doenças - CID, aos fatores externos ao organismo humano que provocam lesões, envenenamentos ou efeitos adversos ao homem), que são hoje as principais causas de adoecimento e morte da população (World Bank, 1989; Ramos et al., 1987).

A magnitude da mortalidade na população tem sido, até aqui, prioritariamente descrita por meio das taxas de mortalidade e da mortalidade 
proporcional. Sob estes pontos de vista, as doenças do aparelho cardiovascular são as principais causas de óbito na população em geral, seguidas das causas externas e das neoplasias (Szwarcwald, 1986). No entanto, a esta ótica é pertinente a crítica feita por Gardner \& Sanborn (1990), segundo a qual as taxas brutas e específicas de mortalidade descrevem o quantitativo das mortes na população, mas falham em quantificar o peso resultante desta perda para a sociedade. Neste sentido, têm sido enfatizadas a importância da mortalidade prematura como expressão do valor social da morte e a necessidade de operacionalizar sua medida e análise (Romeder \& McWhinnie, 1977; Silva, 1984; Rodriguez \& Motta, 1989). A morte, quando ocorre numa etapa da vida de altas criatividade e produtividade, não só pune o próprio indivíduo e o grupo que lhe é próximo, mas também priva a coletividade de seu potencial econômico e intelectual. $\mathrm{O}$ indicador "anos potenciais de vida perdidos" (APVP), que explicita o total de anos de vida em potencial perdidos a cada óbito, qualifica esta morte.

Neste estudo enfoca-se o Rio de Janeiro, pelo fato de tanto o estado quanto sua capital serem típicos de um padrão de mortalidade encontrado nos estados do Sul e Sudeste, e muito possivelmente na maioria dos estados da Federação num futuro próximo. A associação entre urbanização e tipo de padrão epidemiológico existente fortalece a suposição de que a análise da situação de saúde no Rio de Janeiro pode contribuir para expor fases mais avançadas do processo de transição epidemiológica brasileiro. As taxas de urbanização no estado do Rio de Janeiro sempre foram as mais altas do Brasil. Ainda que atualmente apresente o mais lento incremento anual de população urbana, o Rio de Janeiro continua sendo o estado mais urbanizado do Brasil, com $91 \%$ de sua população vivendo em cidades (Reichenheim, 1988).

O objetivo deste artigo é redimensionar o perfil de mortalidade sob a ótica do indicador APVP (total e desagregado por sexo), por principais causas de óbito, no estado e no município do Rio de Janeiro em 1990.

\section{METODOLOGIA}

Existem diversas maneiras de calcular o indicador APVP, sendo que a escolha de cada método depende dos aspectos da mortalidade prematura que se procura evidenciar. As divergências entre esses métodos dizem respeito à definição das idades nas quais as perdas sociais e econômicas têm seu início e fim (Gardner \& Sanborn, 1990). No presente estudo utiliza-se o método descrito por Romeder \& McWhinnie (1977). Trata-se de um método simples e de fácil entendimento, permitindo o seu uso de forma sistemática no planejamento em saúde.

Tendo em vista uma expectativa de vida ao nascer de 70 anos, multiplica-se o número de óbitos ocorridos em cada idade pelo número de anos que faltam para alcançar aquele limite. A seguir soma-se cada um dos subtotais. Desta forma, as causas de morte que tendem a se concentrar nas faixas etárias mais novas apresentarão um quantitativo de APVP maior em relação àquelas que incidem mais nos últimos anos da vida.

Matematicamente, a expressão de APVP é a seguinte:

APVP $=\sum_{i=1}^{69} a_{i} d_{i}=\sum_{i=1}^{69}(70-i-0,5) d_{i}$

onde

$\mathrm{a}_{\mathrm{i}}=$ número de anos que faltam para completar 70 anos, quando a morte ocorre entre as idades de i e i+1 anos;

$\mathrm{d}_{\mathrm{i}}=$ número de óbitos ocorridos entre as idades de i e i+1 anos, empregando-se o ajuste de 0,5 quando se arbitra que todas as mortes ocorreram no meio do ano.

Tomam-se como referência as faixas etárias e as médias do correspondente número de anos que faltam para completar 70 anos, quando a morte ocorre entre as idades de i e i+1 anos, e o número de óbitos ocorridos entre as idades de i e i+1 anos.

Para uma comparação entre populações de tamanhos e pirâmides populacionais diferentes são utilizadas, respectivamente, a taxa de APVP (TAPVP) e a taxa de APVP padronizada (TAPVPP), cujas expressões matemáticas são as seguintes: 


$$
\begin{aligned}
& \text { TAPVP }=\sum_{i=1}^{69} a_{i} d_{i} \cdot 1000 / N \\
& \text { onde } \\
& \begin{array}{l}
\mathrm{N}= \\
\text { número de pessoas entre } 1 \text { e } 70 \text { anos de idade } \\
\text { na população real; }
\end{array}
\end{aligned}
$$

Obs.: $\mathrm{a}^{1}$ e d ${ }^{1}$ já se encontram definidos acima.

$$
T A P V P P=\sum_{i=1}^{69} a_{i}\left(d_{i} / p_{i}\right) \cdot\left(p_{i r} / N_{r}\right) \cdot 1000
$$

Onde

$\mathrm{p}^{\mathrm{i}}=$ número de pessoas na idade $i$ na população real.

$\mathrm{p}_{\mathrm{ir}}=$ número de pessoas na idade $i$ na população de referência;

$\mathrm{N}_{\mathrm{r}}=$ número de pessoas entre as idades de 1 e 70 anos na população de referência.

Obs.: $\mathrm{a}_{\mathrm{i}}, \mathrm{d}_{\mathrm{i}}$ e $\mathrm{N}$ já se encontram definidos acima.

As populações do estado e do município do Rio de Janeiro foram obtidas a partir das tabulações preliminares do Censo Demográfico brasileiro de 1991, enquanto os dados de mortalidade foram obtidos na Secretaria de Estado de Saúde do Rio de Janeiro. Utilizou-se a soma das duas populações como referência para a padronização das taxas.

\section{RESULTADOS}

As Tabelas 1 e 2 apresentam o total de APVP e as taxas de APVP brutas e padronizadas por grandes grupos de causas e sexo para o estado e o município do Rio de Janeiro, respectivamente, em 1990.

Nota-se que o grupo das causas externas (traumas e violências) é responsável por mais de 1/3 dos APVPs, seguido pelo grupo das doenças cardiovasculares e pelo grupo das neoplasias. Estes três grupos são responsáveis por quase $70 \%$ de todos os APVPs nas duas regiões.

As Tabelas 1 e 2 mostram, também, que, de modo geral, o perfil de APVP proporcional pouco se modifica ao se comparar o estado com o município do Rio de Janeiro. Percebe-se, entretanto, que as taxas para o município do Rio de Janeiro são quase sempre menores, exceção feita às neoplasias. Uma possível explicação para tal fato poderia ser uma distribuição etária diferente entre as duas populações. Todavia, isto não é corroborado quando se calcula as taxas de APVP padronizadas por idade.

Desagregando-se as taxas por sexo, dois pontos emergem. Primeiro, as taxas de APVP para ambos os sexos são menores no município do Rio de Janeiro, exceção feita, novamente, às neoplasias. Segundo, o total de APVP e as taxas de APVP são, como um todo, marcadamente mais altos no sexo masculino. Esta discrepância deve-se, principalmente, à ação das causas externas. Embora, para todas as causas, o sexo masculino sempre apresente taxas de APVP maiores que o sexo feminino, a exclusão do efeito das causas externas na mortalidade masculina é suficiente para tornar as taxas de APVP total entre os sexos bastante semelhantes. As Figuras 1 e 2 ilustram mais claramente estas discrepâncias.

Pode-se avaliar o impacto real dos traumas e violências nas perdas de anos potenciais de vida calculando-se a distribuição dos APVPs por causas específicas nas faixas etárias de 1 a 29 anos. Cerca de $40 \%$ dos APVPs em ambas as regiões são devidos a mortes nesta faixa etária, embora menos de $20 \%$ dos óbitos entre 1 e 69 anos ocorram neste período da vida. $\mathrm{O}$ fato de as causas externas serem responsáveis por cerca de $63 \%$ dos óbitos nesta faixa etária ressalta a sua importância como fator de perda de anos potenciais de vida na população.

As Tabelas 3 e 4 mostram os APVPs e as taxas de APVP pelas principais causas específicas de óbito acima de 1 ano de idade para o estado e para o município do Rio de Janeiro, respectivamente, no ano de 1990. As últimas colunas (posições I, II e III) referem-se às posições ocupadas por cada causa específica quando se obtém, respectivamente, o perfil de APVP em 1990; o perfil de mortalidade através das taxas de mortalidade geral, excluídas as mortes em menores de 1 ano; e o perfil de APVP em 1985. 
O perfil de APVP difere de maneira discreta entre as localidades estudadas, a maior diferença sendo observadas nos "outros tipos de violências". Nota-se que a análise dos APVPs coloca os homicídios e os traumas causados por veículos automotores (TCVA) em maior destaque do que quando se faz uma análise tradicional, através das taxas de mortalidade.

Quando se compara o perfil dos APVPs por causas específicas em 1985 e 1990, percebemse duas importantes modificações. Primeiro, há crescimento da participação dos homicídios, passando estes a ser a principal causa de APVP tanto no estado quanto no município. Segundo, observa-se um decréscimo da participação dos "outros tipos de violências". Pode-se sugerir que parcela desta queda seja devida a uma melhoria na classificação das causas externas. Os TCVA também sobem de posição, possivelmente devido a esta melhor classificação. A este argumento soma-se o fato de que ocorreu um aumento real no total e nas taxas de APVP por causas externas (Werneck \& Reichenheim, 1992). Pode-se apreender, ainda, que essas mudanças têm magnitude diferente nas duas regiões avaliadas, sendo o aumento dos homicídios maior no município. Este aumento leva a taxa de APVP por homicídios no município a um patamar semelhante ao do estado.

TABELA 1. Anos Potenciais de Vida Perdidos (APVP) por Grandes Grupos de Causas e Sexo, Estado do Rio de Janeiro, 1990

\begin{tabular}{|c|c|c|c|c|c|}
\hline \multirow{3}{*}{ Causas de Morte } & \multirow{3}{*}{ Sexo } & \multicolumn{2}{|c|}{ APVP Bruto } & \multicolumn{2}{|c|}{ Taxas de APVP } \\
\hline & & & & & \\
\hline & & $\mathrm{N}^{\mathrm{o}}$ & $\%$ & Bruta & Padronizada \\
\hline \multirow[t]{3}{*}{ Causas Externas } & M & $489.775,5$ & 48,4 & 84,3 & 84,1 \\
\hline & $\mathrm{F}$ & $67.774,0$ & 16,5 & 11,0 & 10,9 \\
\hline & $\mathrm{T}$ & $557.549,5$ & 39,2 & 46,6 & 46,4 \\
\hline \multirow[t]{3}{*}{ Doenças do Aparelho Cardiovascular } & M & $165.773,5$ & 16,4 & 78,5 & 79,3 \\
\hline & $\mathrm{F}$ & 113.316 .5 & 27,5 & 18,4 & 19,0 \\
\hline & $\mathrm{T}$ & $279.040,0$ & 19,6 & 23,3 & 24,0 \\
\hline \multirow[t]{3}{*}{ Doenças Neoplásicas } & M & $64.884,5$ & 6,4 & 11,1 & 11,4 \\
\hline & $\mathrm{F}$ & $64.501,0$ & 15,7 & 10,5 & 10,7 \\
\hline & $\mathrm{T}$ & $129.385,5$ & 9,1 & 10,8 & 11,1 \\
\hline \multirow[t]{3}{*}{ Doenças do Aparelho Respiratório } & M & $60.906,0$ & 6,0 & 10,5 & 10,6 \\
\hline & $\mathrm{F}$ & $37.844,0$ & 9,2 & 6,1 & 6,1 \\
\hline & $\mathrm{T}$ & $98.750,0$ & 6,9 & 8,2 & 8,3 \\
\hline \multirow[t]{3}{*}{ Doenças do Aparelho Digestivo } & M & $47.796,0$ & 4,7 & 8,2 & 8,4 \\
\hline & $\mathrm{F}$ & $15.566,0$ & 3,8 & 2,5 & 2,6 \\
\hline & $\mathrm{T}$ & $63.362,0$ & 4,5 & 5,3 & 5,4 \\
\hline \multirow[t]{3}{*}{ Doenças Infecciosas e Parasitárias } & M & $41.441,5$ & 4,1 & 7,1 & 7,2 \\
\hline & $\mathrm{F}$ & $21.632,0$ & 5,3 & 3,5 & 3,5 \\
\hline & $\mathrm{T}$ & $63.073,5$ & 4,4 & 5,3 & 5,3 \\
\hline \multirow[t]{3}{*}{ Outras Causas } & M & $141.601,5$ & 14,0 & 24,4 & 24,6 \\
\hline & $\mathrm{F}$ & $90.837,0$ & 22,1 & 14,7 & 18,9 \\
\hline & $\mathrm{T}$ & $232.438,5$ & 16,3 & 19,4 & 19,6 \\
\hline \multirow[t]{3}{*}{ Total } & M & $1.012 .128,5$ & 100,0 & 174,2 & 175,5 \\
\hline & $\mathrm{F}$ & $411.470,5$ & 100,0 & 66,8 & 67,7 \\
\hline & $\mathrm{T}$ & $1.423 .599,0$ & 100,0 & 118,9 & 120,1 \\
\hline
\end{tabular}


TABELA 2. Anos Potenciais de Vida Perdidos (APVPs) por Grandes Grupos de Causas e Sexo, Município do Rio de Janeiro, 1990

\begin{tabular}{|c|c|c|c|c|c|}
\hline \multirow[b]{2}{*}{ Causas de Morte } & \multirow[b]{2}{*}{ Sexo } & \multicolumn{2}{|c|}{ APVP Bruto } & \multicolumn{2}{|c|}{ Taxas de APVP } \\
\hline & & $\mathrm{N}^{\mathrm{o}}$ & $\%$ & Bruta & Padronizada \\
\hline \multirow[t]{3}{*}{ Causas Externas } & M & $186.800,5$ & 45,3 & 76,7 & 77,3 \\
\hline & $\mathrm{F}$ & $27.245,5$ & 16,1 & 10,2 & 10,3 \\
\hline & $\mathrm{T}$ & $214.046,0$ & 36,8 & 41,8 & 42,4 \\
\hline \multirow[t]{3}{*}{ Doenças do Aparelho Cardiovascular } & M & $68.200,0$ & 16,5 & 28,0 & 26,4 \\
\hline & $\mathrm{F}$ & $46.816,5$ & 27,7 & 17,4 & 16,3 \\
\hline & $\mathrm{T}$ & $115.016,5$ & 19,8 & 22,5 & 21,0 \\
\hline \multirow[t]{3}{*}{ Doenças Neoplásicas } & M & $31.018,0$ & 7,5 & 12,7 & 12,1 \\
\hline & $\mathrm{F}$ & $31.747,5$ & 18,8 & 11,8 & 11,2 \\
\hline & $\mathrm{T}$ & $62.765,5$ & 10,8 & 12,3 & 11,6 \\
\hline \multirow[t]{3}{*}{ Doenças do Aparelho Respiratório } & M & $23.333,0$ & 5,7 & 9,6 & 9,4 \\
\hline & $\mathrm{F}$ & $13.579,5$ & 8,0 & 5,1 & 5,0 \\
\hline & $\mathrm{T}$ & $36.912,5$ & 6,4 & 7,2 & 7,1 \\
\hline \multirow[t]{3}{*}{ Doenças do Aparelho Digestivo } & M & $20.294,5$ & 4,9 & 8,3 & 8,0 \\
\hline & $\mathrm{F}$ & $5.926,0$ & 3,5 & 2,2 & 2,1 \\
\hline & $\mathrm{T}$ & $26.220,5$ & 4,5 & 5,1 & 4,9 \\
\hline \multirow[t]{3}{*}{ Doenças Infecciosas e Parasitárias } & M & $16.898,5$ & 4,1 & 6,9 & 6,9 \\
\hline & $\mathrm{F}$ & $8.282,0$ & 4,9 & 3,1 & 3,1 \\
\hline & $\mathrm{T}$ & $25.180,5$ & 4,3 & 4,9 & 4,9 \\
\hline \multirow[t]{3}{*}{ Outras Causas } & M & $65.544,0$ & 15,9 & 26,9 & 26,4 \\
\hline & $\mathrm{F}$ & $35.268,5$ & 20,9 & 13,1 & 13,0 \\
\hline & $\mathrm{T}$ & $100.812,5$ & 17,4 & 19,7 & 19,4 \\
\hline \multirow[t]{3}{*}{ Total } & $\mathrm{M}$ & $412.088,5$ & 100,0 & 169,2 & 166,3 \\
\hline & $\mathrm{F}$ & $168.865,5$ & 100,0 & 169,2 & 166,3 \\
\hline & $\mathrm{T}$ & $580.954,0$ & 100,0 & 113,5 & 111,3 \\
\hline
\end{tabular}

FIGURA 1. Anos Potenciais de Vida Perdidos Segundo Grupos de Causas e Sexo no Estado do Rio de Janeiro

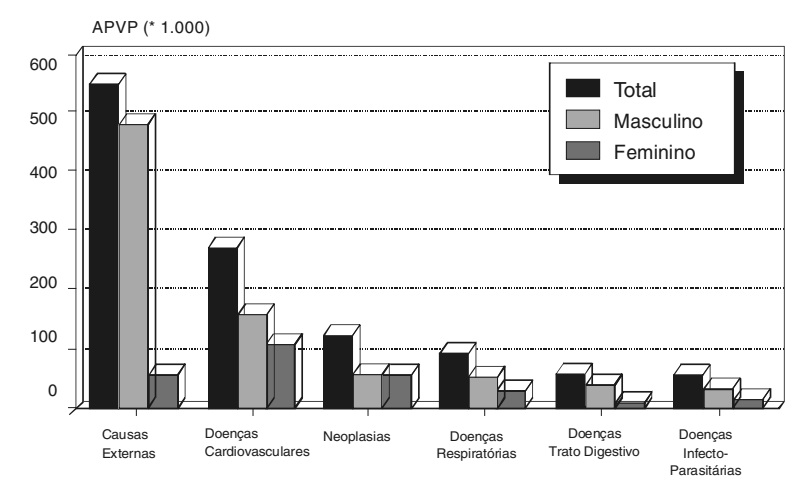


FIGURA 2. Anos Potenciais de Vida Perdidos Segundo Grupos de Causas e Sexo no Município do Rio de Janeiro

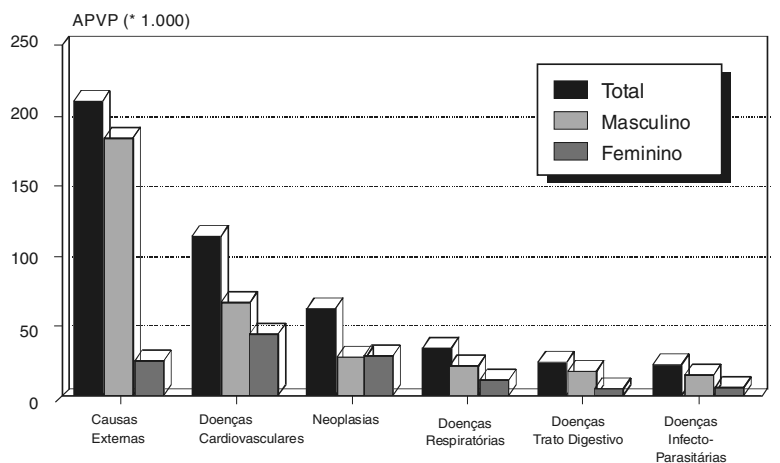

\section{DISCUSSÃO}

A descrição da situação de saúde da população brasileira por meio dos indicadores tradicionais tem se tornado cada vez mais insuficiente. Ainda que limitados, os indicadores de mortalidade desempenham um papel importante na apreensão desta realidade, especialmente ao incorporarem informações acerca do impacto social e econômico da morte. As taxas de mortalidade descrevem o quantitativo das mortes na população, mas não conseguem atribuir um valor a esta perda para a sociedade.

$\mathrm{O}$ indicador de mortalidade prematura — "anos potenciais de vida perdidos" — é uma boa alternativa para esta finalidade.

Dois pontos serão objeto de discussão: primeiro abordam-se os limites do método utilizado; a seguir interpretam-se os resultados obtidos.

\section{Limites do Método Utilizado e Erros Potenciais}

A escolha do melhor indicador de mortalidade prematura é um problema a ser resolvido. A incorporação dos óbitos infantis, por exemplo, modifica profundamente o perfil de APVP, e esta prática vem sendo adotada desde 1986 nos relatórios publicados pelo Center of Disease Control — CDC (Gardner \& Sanborn, 1990). Alguns autores têm centrado sua atenção nos anos potenciais de vida perdidos na faixa etária acima de 15 anos, priorizando as perdas econômicas ligadas ao período de produção material

(Gardner \& Sanborn, 1990). Esta opção refere- se, em última análise, aos objetivos do estudo e deve estar explícita.

A construção e utilização do APVP levam em consideração alguns pontos que merecem elaboração. Uma questão decorre justamente do fato de não serem considerados os óbitos ocorridos em crianças abaixo de 1 ano de idade. Assim, as pneumonias, bem como aquelas doenças cujo impacto na mortalidade é mais intenso nesta faixa etária, são subestimadas pelo uso deste método. Pode-se ponderar, no entanto, que nesta idade as mortes devem-se a causas muito específicas, e sua decorrente supervalorização poderia não ter respaldo social. Citando Romeder \& McWhinnie (1977), " a mortalidade de crianças menores de um ano de idade é menos perturbadora que a mortalidade de crianças com mais idade e a dos adultos". Além do mais, existem bons indicadores para a qualificação da morte em menores de 1 ano, tais como os coeficientes de mortalidade infantil, neonatal e perinatal, entre outros.

Podem ocorrer erros de estimação ao se arbitrar uma expectativa de vida de 70 anos. Primeiro, por não ser este limite homogêneo entre os sexos, superestimam-se os APVPs no sexo masculino. Segundo, para o Brasil, onde a expectativa de vida é de 66 anos (Unicef, 1992), este limite é exagerado, também levando a uma superestimação. Neste estudo optou-se por sua utilização, para facilitar a comparação externa dos dados e por questões operacionais, visto que as estatísticas de mortalidade do Ministério da Saúde apresentam seus dados agrupados por faixa etária com intervalos de 10 anos, a partir dos 20 anos de idade (MS, 1992). 
Terceiro, por não estarem sendo considerados os efeitos de coorte, devido ao fato de a população que morre atualmente por uma determinada causa ter uma expectativa de vida menor que esta a qual se está inferindo, superestimam-se os APVPs. Por outro lado, subestima-se a importância da mortalidade prematura, na medida em que o efeito de coorte é mais intenso nas idades mais avançadas.

Uma outra questão que merece comentário é que a eliminação de uma determinada causa de morte não implica necessariamente uma sobrevivência até o limite esperado, havendo a possibilidade de morrer por outras causas evidentemente não computadas. Isto pode levar a uma identificação espúria do impacto que esta morte evitada teria, na medida em que um indivíduo pode, na realidade, não ter todos aqueles anos potenciais de vida ganhos.

Um cálculo de APVP que levasse em conta todas essas considerações tornar-se-ia demasiado complexo e de utilidade restrita para o planejamento em saúde. Esses problemas já foram levantados na literatura e a sua correção tem sido questionada (Romeder \& McWhinnie, 1977).

TABELA 3. Anos Potenciais de Vida Perdidos (APVPs) por Causas Específicas, Estado do Rio de Janeiro, 1990

\begin{tabular}{|c|c|c|c|c|c|c|c|}
\hline \multirow[b]{2}{*}{ Causas de Morte } & \multicolumn{2}{|c|}{ APVP Bruto } & \multicolumn{2}{|c|}{ Taxas de APVP } & \multicolumn{3}{|c|}{ Posição } \\
\hline & $\mathrm{N} \%$ & $\%$ & Bruta & Padronizada & I & II & III \\
\hline Homicídios (E55) & $258.676,5$ & 18,2 & 21,6 & 21,5 & $1^{\mathrm{o}}$ & $4^{\circ}$ & $5^{\circ}$ \\
\hline $\begin{array}{l}\text { Outros Tipos de Violência } \\
\text { (E54/E56) }\end{array}$ & $124.563,0$ & 8,7 & 10,4 & 10,4 & $2^{o}$ & $5^{\circ}$ & $1^{\circ}$ \\
\hline $\begin{array}{l}\text { Neoplasmas Malignos } \\
(08-14)\end{array}$ & $123.532,5$ & 8,7 & 10,3 & 10,6 & $3^{\circ}$ & $3^{\circ}$ & $2^{\circ}$ \\
\hline $\begin{array}{l}\text { Acidentes de Trânsito } \\
\text { (E471) }\end{array}$ & $107.687,5$ & 7,6 & 9,0 & 9,0 & $4^{\circ}$ & $6^{\circ}$ & $6^{\circ}$ \\
\hline Doença Cerebrovascular (29) & $98.044,0$ & 6,9 & 8,2 & 8,4 & $5^{\circ}$ & $1^{\mathrm{o}}$ & $3^{\circ}$ \\
\hline $\begin{array}{l}\text { Doença Isquêmica } \\
\text { de Coração ( } 27)\end{array}$ & $91.447,5$ & 6,4 & 7,6 & 7,9 & $6^{\mathrm{o}}$ & $2^{\circ}$ & $4^{\circ}$ \\
\hline $\begin{array}{l}\text { Outros Acidentes } \\
\text { (E47-E53/EXC.E471) }\end{array}$ & $66.622,5$ & 4,7 & 5,6 & 5,5 & $7^{\circ}$ & $10^{\circ}$ & $7^{\circ}$ \\
\hline Pneumonia (321) & $46.182,0$ & 3,2 & 3,9 & 3,8 & $8^{\circ}$ & $7^{\circ}$ & $8^{\circ}$ \\
\hline $\begin{array}{l}\text { Doença Crônica do Fígado/ } \\
\text { Cirrose (347) }\end{array}$ & $31.307,5$ & 2,2 & 2,6 & 2,7 & $9^{\circ}$ & $11^{\circ}$ & $10^{\circ}$ \\
\hline Diabetes Mellitus (181) & $26.374,0$ & 1,9 & 2,2 & 2,3 & $10^{\circ}$ & $8^{\circ}$ & $9^{\circ}$ \\
\hline Doença Hipertensiva (26) & $26.300,0$ & 1,8 & 2,2 & 2,3 & $11^{\circ}$ & $9^{\circ}$ & $11^{\circ}$ \\
\hline Total Parcial & $1.000 .737,0$ & 70,3 & 83,6 & 84,4 & - & - & - \\
\hline Outros & $422.862,0$ & 29,7 & 35,3 & 35,7 & - & - & - \\
\hline Total Geral & $1.423 .599,0$ & 100,0 & 118,9 & 120,1 & - & - & - \\
\hline
\end{tabular}


TABELA 4. Anos Potenciais de Vida Perdidos (APVPs) Por Causas Específicas, Município do Rio de Janeiro, 1990

\begin{tabular}{lccccccc}
\hline \hline & \multicolumn{2}{c}{ APVP Bruto } & \multicolumn{2}{c}{ Taxas de APVP } & \multicolumn{3}{c}{ Posição } \\
\cline { 2 - 7 } Causas de Morte & N\% & $\%$ & Bruta & Padronizada & I & II & III \\
\hline Homicídios (E55) & $110.592,5$ & 19,0 & 21,6 & 22,0 & $1^{\circ}$ & $4^{\circ}$ & $8^{\circ}$ \\
Neoplasmas Malignos (08-14) & $59.985,5$ & 10,3 & 11,7 & 11,1 & $2^{\circ}$ & $1^{\circ}$ & $2^{\circ}$ \\
Acidentes de Trânsito (E471) & $48.015,0$ & 8,3 & 9,4 & 9,5 & $3^{\circ}$ & $7^{\circ}$ & $5^{\circ}$ \\
Doença Isquêmica do Coração (27) & $41.795,0$ & 7,2 & 8,2 & 7,6 & $4^{\circ}$ & $2^{\circ}$ & $3^{\circ}$ \\
Doença Cerebrovascular (29) & $38.758,5$ & 6,7 & 7,6 & 7,1 & $5^{\circ}$ & $3^{\circ}$ & $4^{\circ}$ \\
Outros Tipos de Violência & & & & & & & \\
(E54/E56) & $31.529,0$ & 5,4 & 6,2 & 6,2 & $6^{\circ}$ & $10^{\circ}$ & $1^{\circ}$ \\
Outros Acidentes & & & & & & & \\
(E47-E53/EXC.E471) & $23.909,5$ & 4,1 & 4,7 & 4,7 & $7^{\circ}$ & $10^{\circ}$ & $7^{\circ}$ \\
Pneumonia (321) & $18.560,5$ & 3,2 & 3,6 & 3,6 & $8^{\circ}$ & $5^{\circ}$ & $7^{\circ}$ \\
Doença Crônica do Fígado/Cirrose & & & & & & & \\
(347) & $13.797,5$ & 2,4 & 2,7 & 2,6 & $9^{\circ}$ & $11^{\circ}$ & $9^{\circ}$ \\
Doença Hipertensiva (26) & $10.835,0$ & 1,9 & 2,1 & 2,0 & $10^{\circ}$ & $8^{\circ}$ & $10^{\circ}$ \\
Diabetes Mellitus (181) & $10.595,0$ & 1,8 & 2,1 & 1,9 & $11^{\circ}$ & $6^{\circ}$ & $11^{\circ}$ \\
\hline Total Parcial & $408.373,0$ & 70,3 & 79,8 & 78,2 & - & - & - \\
Outros & $172.581,0$ & 29,7 & 33,7 & 33,1 & - & - & - \\
\hline Total Geral & $580.954,0$ & 100,0 & 113,5 & 111,3 & - & - & - \\
\hline \hline
\end{tabular}

É preciso salientar, também, que a magnitude das causas externas vista através dos APVPs seria ainda mais marcante se os "outros tipos de violência" fossem melhor definidos. Cerca de $80 \%$ destes se referem às lesões que não se sabe se inflingidas intencionalmente ou não, e apenas $20 \%$ são suicídios. Apesar da melhoria detectada na classificação destes óbitos em relação ao ano de 1985, ainda uma boa parcela permanece mal-definida. Grande parte dos "outros tipos de violência" são TCVAs e homicídios mal-classificados (Radis, 1990).

\section{Apreciação dos Resultados}

A população do estado do Rio de Janeiro apresenta um maior risco de perder anos de vida em potencial, em decorrência, principalmente, da ação das causas externas. Esta diferença se mantém para ambos os sexos. Tal resultado poderia ser ainda mais significativo se o município do Rio de Janeiro fosse excluído deste universo.
As causas externas são as principais causas de mortalidade prematura em todo o Rio de Janeiro, sendo responsáveis por $1 / 3$ de todos os APVPs nas duas regiões analisadas. Identificouse que esta participação maciça das causas externas nas perdas de anos potenciais de vida é decorrente de seu impacto preferencial no sexo masculino. As causas externas são responsáveis por cerca de $45 \%$ dos APVPs neste sexo, correspondendo a quase três vezes os APVPs por doenças do aparelho cardiovascular. No sexo feminino, esta proporção é de somente $16 \%$, cerca da metade dos APVPs por doenças do aparelho cardiovascular.

$\mathrm{O}$ redimensionamento da magnitude das causas específicas de morte através deste método mostra uma emergência de todos os tipos de causas externas e uma queda de todas as doenças do aparelho cardiovascular. Estas últimas, quando estudadas através das taxas de mortalidade e mortalidade proporcional, aparecem como principal causa de mortalidade na população. Os achados estão de acordo com os 
de outros estudos (Romeder \& McWhinnie, 1977; Silva, 1984; Arca et al., 1988; Plaut \& Roberts, 1989; Rodriguez \& Motta, 1989).

Nota-se o papel relevante desempenhado pelas violências (homicídios, suicídios e violências não-especificadas) na determinação da mortalidade prematura, o que difere bastante dos países desenvolvidos, onde os TCVAs aparecem como fator principal de APVP (Arca et al., 1988; Plaut \& Roberts, 1989; Rodriguez \& Motta, 1989). Vale apontar que os níveis de APVP encontrados (Tabela 1) são bem superiores aos da maioria dos países do continente americano (Paho, 1986; Smith \& Barss, 1991). As taxas no estado do Rio de Janeiro somente se equiparam às de El Salvador e Guatemala, países ainda há pouco tempo em estado de guerra civil.

A análise deste perfil merece ser contextualizada nos processos sociais vividos no Rio de Janeiro nos últimos anos. Em sociedades desenvolvidas observou-se o que tem sido denominado "transição epidemiológica", a qual se caracteriza pela elevação dos índices de morbi-mortalidade por doenças relacionadas à industrialização e à urbanização, associada à superação das doenças próprias do subdesenvolvimento. Três perfis epidemiológicos contíguos e encadeados são identificáveis. O perfil do primeiro período caracteriza-se pelo predomínio de doenças associadas à pobreza, pela ausência de adequado saneamento ambiental e pelo precário desenvolvimento sócio-econômico. Predominam aqui as doenças infecto-contagiosas. Com as progressivas conquistas sociais, identificadas mais diretamente através da melhoria das condições básicas de vida - alimentação e nutrição, habitação e saneamento, vestuário, transporte - e com os avanços no campo técnico-científico, registrou-se uma transição para um segundo perfil epidemiológico, caracterizado, predominantemente, pelas doenças crônico-degenerativas. Mais recentemente surge um terceiro perfil, marcadamente urbano, caracterizado pelas doenças relacionadas à contaminação/poluição ambiental, pelos traumas intencionais e não-intencionais, pelas afecções psicoemocionais, pela drogadição, etc. (Rossi-Espagnet, 1984; Harpham et al., 1988; Reichenheim \& Werneck, 1992).

Do ponto de vista agregado, ainda que de forma parcial e incompleta, este modelo de transição também tem sido observado no Brasil, principalmete nos grandes centros urbanos. $\mathrm{O}$ Brasil já é um país de população majoritariamente urbana. O crescimento e a consolidação do espaço urbano, enquanto tendência demográfica, são irreversíveis. Em 1980, cerca de 68\% da população brasileira já estava abrigada nas áreas urbanas do país. Para o Brasil como um todo, estimativas prevêem que, em 30 anos, mais de $85 \%$ da população estará vivendo em centros urbanos. O estado do Rio de Janeiro é paradigmático deste processo: no final da década de 80 tinha cerca de $92 \%$ de sua população concentrada em áreas urbanas, $80 \%$ desta somente na Região Metropolitana (Oliveira, 1991).

Antes da década de 80, principalmente nos centros urbanos, observou-se uma tendência à diminuição das doenças infecciosas e parasitárias, enquanto as doenças cardiovasculares, as neoplasias, as doenças mentais e os traumas/ violências cresceram aceleradamente. Na década de 80, estes últimos consolidaram-se como carros-chefes das causas de morbi-mortalidade. Vale ressaltar que é especificamente nesta década que sedimenta-se o advento da terceira fase da transição epidemiológica. Prosseguem o declínio da mortalidade por doenças infecciosas, a estabilidade da mortalidade por doenças cardiovasculares e o aumento da mortalidade por causas externas e neoplasias.

Nesta terceira fase da transição epidemiológica afloram demandas para a solução de problemas de outra ordem que afetam o modo de viver da população. Os traumas, os problemas psico-emocionais e, ainda, a questão da poluição ambiental são alguns exemplos. O que integra estes problemas é o fato de eles se fazerem presentes no cotidiano, e não somente na sua expressão nosológica final. Tanto nos homicídios como nos agravos psico-emocionais chama a atenção não somente o desfecho enquanto magnitude, mas todo o processo de deterioração dos aspectos de qualidade da vida que os acompanham (Reichenheim \& Werneck, 1994).

No caso dos homicídios, o crescimento das taxas de mortalidade é acompanhado também por um crescente impacto nas idades mais jovens, salientando ainda mais este aspecto 
nefasto do processo de transição epidemiológica. Os resultados deste estudo, confrontados com avaliação anterior, mostram que de 1985 a 1990, no estado do Rio de Janeiro, as taxas de anos potenciais de vida perdidos por homicídios subiram de 7,8/1.000 para 21,0/1.000, passando a ocupar o primeiro lugar entre as causas de APVP. Este dado possivelmente representa apenas a ponta de um iceberg. A violência crescente, posta em evidência no Rio de Janeiro, transforma o cotidiano em um verdadeiro exercício de sobrevivência, modalizado por tensões e medos.

O enfrentamento destes problemas implica uma absorção de novos conhecimentos e a elaboração de estratégias bastante diferenciadas de atuação. Torna-se cada vez mais necessária a introdução de novas metodologias que permitam a definiçãa de "prioridades dentro de prioridades".

A análise destes problemas através do método aqui apresentado colocou as causas externas no centro desta discussão. A incorporação da noção de valor social da morte (como é o caso da morte prematura) acrescenta mais um dado à definição das prioridades na área da saúde e vem de encontro com as manifestações, cada vez mais freqüentes nos meios de comunicação de massa, clamando por um enfrentamento mais incisivo e eficaz do problema das violências e traumas. Soma-se a estes fatos o aspecto das reais possibilidades de intervenção. A prevenção dos TCVAs e homicídios, por exemplo, deve considerar, também, a utilização de estratégias de ação passivas e legais (justiça retributiva), que não implicam modificações de hábitos e costumes individuais (Haddon \& Baker, 1981; Coelho, 1988). A prevenção das doenças cardiovasculares e das neoplasias, ao contrário, implica uma ênfase das ações de saúde pública nas mudanças a nível dos comportamentos individuais para o alcance de resultados concretos (World Bank, 1989; Avis et al., 1990).

Reflexos de um processo de urbanização desorganizado e excludente, os conflitos sociais urbanos manifestam-se por diversas vias, sendo a violência seu retrato mais cruel. $\mathrm{O}$ grande impacto dos homicídios e dos TCVAs no perfil de mortalidade prematura reafirma a sua impor- tância como "problema de saúde pública" e salienta a definitiva necessidade de comprometimento do Estado e da sociedade civil na busca de soluções para o mesmo.

\section{RESUMO}

REICHENHEIM, M. E. \& WERNECK, G. L. Anos Potenciais de Vida Perdidos no Rio de Janeiro, 1990. As Mortes Violentas em Questão. Cad. Saúde Públ., Rio de Janeiro, 10 (suplemento 1): 188-198, 1994.

Neste artigo faz-se uma análise da magnitude das causas de morte no estado e no município do Rio de Janeiro em 1990, com base no cálculo dos "anos potenciais de vida perdidos" (APVPs) entre as idades de 1 e 70 anos. Esta análise leva a uma substancial modificação na importância relativa das causas de óbito, pois este método imputa um maior valor às mortes que ocorrem nas fases precoces da vida.

De acordo com este método, a análise pelos grandes grupos de causas de óbito mostra que as duas regiões apresentam o grupo das "causas externas" como principal causa de APVP, sendo as mesmas responsáveis por mais de 30,0\% dos APVPs entre 1 e 70 anos. Esta estimativa está em contraposição à predominância das doenças cardiovasculares, indicada pelos perfis de mortalidade que utilizam os coeficientes específicos de mortalidade por grandes grupos de causas. A análise por causas específicas de morte aponta os homicídios como principais responsáveis pelos APVPs nas duas regiões, seguidas pelos neoplasmas malignos, no município do Rio de Janeiro, e por "outros tipos de violência" (suicídios e violências em que ignora se de causa acidental ou intencional), no estado do Rio de Janeiro.

Exceção feita às doenças neoplásicas, as taxas de APVP por grandes grupos de causas, em ambos os sexos, são sempre menores no município do Rio de Janeiro. O total de APVP e as taxas de APVP são marcadamente mais altas no sexo masculino, em decorrência, principalmente, das causas externas. Os autores salientam a importância da utilização deste indicador de mortalidade 
prematura para a definição de prioridades e chamam a atenção para o caráter devastador dos traumas e violências como causa de mortes em idades jovens.

Palavras-Chave: Traumas; Violência; Acidentes; Suicídio

\section{REFERÊNCIAS BIBLIOGRÁFICAS}

ARCA, M.; DI ORIO, F.; FORASTIENE, F.; TASCO, C. \& PERUCCI, C.A, 1988. Years of potential life lost (YPLL) before age 65 in Italy. American Fournal Public Health, 78: 1202-1205.

AVIS, N. E.; MCKINLAY, J. B. \& SMITH, K. W., 1990. Is cardiovascular risk factor knowledge sufficient to influence behavior? American Fournal of Preventive Medicine, 6: 137-144.

COELHO, E. C., 1988. A criminalidade urbana violenta. Dados - Revista de Ciências Sociais, 31: $145-84$.

GARDNER, J. W. \& SANBORN, J. S., 1990. Years of potential life lost (YPLL) - What does it measure? Epidemiology, 1:322-329.

HADDON Jr., W. \& BAKER, S. P., 1981. Injury control. In: Preventive Medicine and Public Health (D. Clark \& M. MacMahon, eds.), $2^{\text {nd }}$ edition, Boston: Little Brown \& Co.

HARPHAM, T.; LUSTY, T. \& VAUGHAN, P., 1988. In the Shadow of the City, Community Health and the Urban Poor. Oxford: Oxford University Press.

MS (Ministério da Saúde), 1992. Estatísticas de Mortalidade, 1987. Brasília: Centro de Processamento de Dados. Brasília, DF: Ministério da Saúde.

OLIVEIRA, J. S., 1991. Desigualdades sociais no Rio. A violência Quotidiana. In: Rio de todas as Crises II, pp.4-16, Rio de Janeiro: IUPERJ. (Série Estudos, 8)

PAHO (Pan American Health Organization), 1986. Health conditions in the Americas 1981-1984, Vol I, Washington, D.C.: PAHO. (Scientific Publication, 500)

PLAUT, R. \& ROBERTS, E., 1989. Preventable mortality: indicator or target? Applications in developing coutries. World Health Statistics Quarterly, 42: 04-15.

RADIS (Reunião Análise e Difusão de Informação sobre Saúde), 1990. Regiões metropolitanas: Violência na vida e na morte. Dados, 14 .

RAMOS, L. R.; VERAS, R. P. \& KALACHE, A., 1987. Envelhecimento populacional: uma realidade brasileira. Revista de Saúde Pública, 21: 211-224.
REICHENHEIM, M. E., 1988. Child Health in an Urban Context: Risk Factors in a Squatter Settlement of Rio de Faneiro. PhD Thesis, London: University of London.

REICHENHEIM, M. E. \& WERNECK, G. L., 1992. A "Malária Urbana", Existe um Adoecer Urbano? Documento elaborado para a Oficina de Trabalho: Saúde e Grandes Cidade: Construção de uma Agenda para Pesquisa, Rio de Janeiro: Instituto de Medicina Social. (Mimeo).

, 1994. Adoecer e morrer no Brasil dos anos 80: perspectivas de novas abordagens. In: Saúde e Sociedade no Brasil na década de 80. (R. F. N. Guimarães \& R. W. Tavares, orgs.), pp. 113-130, Rio de Janeiro: Instituto de Medicina Social/Relume Dumará.

RODRIGUEZ. L. A. G. \& MOTTA, L. C., 1989. Years of potential life lost: application of an indicator for assessing premature mortality in Spain and Portugal. World Health Statistics Quaterly, 42: 50-56.

ROMEDER, J. M. \& McWHINNIE, J. R., 1977. Potential years of life lost between ages 1 and 70: an indicator of premature mortality for health planning. International Fournal of Epidemiology, 6: 143-151.

ROSSI-ESPAGNET, A., 1984. Primary Health Care in Urban Areas: Reaching the Urban Poor in Developing Countries. A state of the art report by UNICEF and WHO, N ${ }^{\circ} 2499 M$. Genebra: WHO. (Mimeo).

SILVA, M. G. C., 1984. Anos potenciais de vida perdidos segundo causas em Fortaleza (Brasil), 1978-1980. Revista de Saúde Pública, 18: 108121.

SMITH, G. \& BARSS, P., 1991. Unintentional injuries in developing countries: the epidemiology of a neglected problem. Epidemiologic Reviews, 13: 228-66.

SZWARCWALD, C. L., 1986. Mortalidade por causas externas no Estado do Rio de Janeiro no período de 1976 a 1980. Cadernos de Saúde Pública, 2: 19-41.

UNICEF (United Nations Children' Fundation), 1992. Situação Mundial da Infância, 1992. Brasília, DF: Unicef.

WERNECK, G. L. \& REICHENHEIM, M. E., 1992. Anos Potenciais de Vida Perdidos no Rio de Faneiro, 1985. As Mortes Violentas em Questão, Rio de Janeiro: IMS/UERJ. (Série Estudos em Saúde Coletiva, 4)

WORLD BANK, 1989. Brazil. Adult Health in Brazil: Ajusting to New Challenges. Washington, D.C.: Brazil Departament, World Bank. 